Rev. Col. Ciencia. Vol. 2, no. 1. Octubre 2020 - marzo 2021. ISSN L 2710-7434 pp. 42-56

\title{
CARACTERIZACIÓN ECOLÓGICA DE LOS CALADEROS DE PESCA EN LA COSTA DE PIXVAE, GOLFO DE CHIRIQUÍ, PACÍFICO PANAMEÑO
}

\section{FISHING GROUNDS CHARACTERIZATION FROM THE PANAMANIAN PACIFIC IN PIXVAE, GULF OF CHIRIQUÍ}

\author{
Luis A. Montes ${ }^{1}$, Ángel J. Vega ${ }^{12}$, Yolani A. Robles P. ${ }^{1}$, Kevan Mantell $^{3}$ \\ ${ }^{1}$ Universidad de Panamá (CCIMBIO-COIBA-UP). Centro de Capacitación, Investigación y Monitoreo de \\ la Biodiversidad en el Parque Nacional Coiba-Universidad de Panamá (CCIMBIO-COIBA-UP); \\ montes lu@hotmail.com, angel.vega@up.ac.pa, yolany.robles@up.ac.pa Panamá \\ ${ }^{2}$ Estación Científica del Parque Nacional Coiba (Coiba-AIP). Panamá \\ 32Dive Base Coiba; uwkevan@gmail.com Panamá
}

\begin{abstract}
RESUMEN
La comunidad de Pixvae es uno de los principales poblados en el área de influencia del Parque Nacional Coiba. Entre marzo-mayo de 2015 y agosto de 2016, se evaluaron las comunidades coralinas asociadas a los caladeros de pesca en la Ensenada de Pixvae, con la finalidad de determinar las relaciones existentes entre la composición del fondo y la presencia de peces conspicuos. Para esto se establecieron transectos de $50 \mathrm{~m}$ de longitud en 12 sitios asociados de pesca y se realizaron censos visuales para conteo de peces, y se grabaron videos en cada transecto. Los resultados indican que los ambientes marinos dominantes son tipo rocosos con promedio de cobertura de $60 \%$, otro $18 \%$ lo componen arena-grava, un $12 \%$ coral duro, algas $8 \%$, coral negro $1 \%$ y octocoral $1 \%$. Los índices de diversidad de Shannon-Weiner ( $\left.H^{\prime}\right)$ en los dos periodos no mostró diferencias significativas. Se reportan 12 especies de coral escleractinio, 4 especies de octocorales y una especie de coral negro. En los peces se registraron 3348 individuos ubicados en 50 especies; la mayor riqueza se presentó en ambientes rocosos de poca profundidad y la mayor abundancia $(>500)$ en ambientes profundos de corales negros. El Índice de Valor Biológico (IVB) indicó que el pargo es un recurso que cuenta con buena presencia en la mayoría de los sitios, lo que ha llevado a presiones en el sistema. Este estudio aporta información científica, con la finalidad de promover herramientas de manejo que garanticen un uso sostenible de los recursos en la Ensenada de Pixvae.
\end{abstract}

PALABRAS CLAVE: Comunidades coralinas, diversidad, abundancia, peces conspicuos, Pixvae

\section{ABSTRACT}

Pixvae is one of the main settlements within the influence area of Coiba National Park. Between March-May 2015 and August 2016, the coralline communities associated with fishing grounds were evaluated in Ensenada de Pixvae with the goal of determining the existing relationships between bottom composition and conspicuous fish presence. In order to achieve this goal, 50meter-long transects were established at 12 sites associated with fishing, where visual censuses for fish counts and video recording of each transect were carried out. The results indicate that rock bottom is the dominant marine habitat with $60 \%$ average cover, another $18 \%$ is composed of gavel-sand, $12 \%$ hard coral, $8 \%$ algae, $1 \%$ black coral and $1 \%$ octocoral. Shannon-Weiner diversity index $\left(\mathrm{H}^{\prime}\right)$ from both research periods did not show significant difference. Twelve scleractinian coral species, 4 octocorals and one species of black coral are reported for this study. A total of 3,348 fish individuals from 50 species were recorded. Highest species richness occurred at shallow rock bottom habitats, whereas highest abundance $(>500)$ was recorded at deeper black coral bottom. Biological value index (IVB) indicated that snapper is a resource with considerable 
presence at most sites, which has generated pressure in the system. This study provides scientific information with the objective to promote management tools that support sustainable use of the resources in Ensenada de Pixvae.

KEYWORDS: Coralline communities, diversity, abundance, conspicuous fish, Pixvae

Artículo recibido: 26 de agosto de 2020.

Artículo aceptado: 27 de septiembre de 2020.

\section{INTRODUCCIÓN}

Las áreas de pesca adyacentes a Áreas Marinas Protegidas (AMP) son sitos de gran interés para los pescadores, por los beneficios que redundan de la conservación como es la renovación de recursos (Roberts et al., 2001), tal es el caso de Pixvae, una de las principales comunidades costeras en el área de influencia del Parque Nacional Coiba (PNC). En este sector se desarrolla una pesca artesanal dirigida a peces de importancia comercial como son los pargos, que representan más del $50 \%$ de las capturas de la pesca artesanal de exportación procedente del Golfo de Chiriquí (Vega et al. 2016a).

Algunos pargos, en ciertas épocas del año, se acercan a la costa en grandes concentraciones, principalmente los pargos seda (Lutjanus peru) y mancha (L. guttatus), debido a las masas de agua fría que se acercan a la costa. Estos avances son aprovechados por las comunidades costeras del área de influencia del PNC, capturándolas con redes de enmalle (trasmallos) como principal arte, y no existen controles de uso en sitios sensitivos como fondos coralinos tanto fuera como dentro de áreas protegidas. La información disponible sobre el estado de los recursos pesqueros del PNC y su zona de influencia, provienen de datos dependientes de las pesquerías y revelan el uso insostenible de los principales recursos pesqueros y la necesidad de implementar estrategias de manejo (Vega et al., 2016a y b).

A nivel de la región se ha documentado el impacto que han tenido las principales pesquerías. En un periodo de 40 a 50 años, la estructura de tallas de las principales especies objetivo ha disminuido considerablemente y la pesquería ha pasado de la captura de grandes peces longevos (depredadores), a pequeños peces con periodos de vida corto (Pauly et. al, 1998; Sala et al, 2004; Salas et al, 2007); acarreando efectos sobre el medio marino y las funciones ecosistémicas de las especies (Ramírez-Ortiz et al., 2017).

Según Roberts (1995), para un manejo costero efectivo se requiere de un entendimiento profundo sobre los efectos de la explotación de los recursos. La relación entre las características de los hábitats y las comunidades de peces ha sido tratada ampliamente en la literatura científica alrededor del mundo y en diversos hábitats (Friedlander \& Parrish, 1997; Ferreira et al., 2000; Gratwicke \& Speight, 2005; Tokeshi \& Arakaki, 2012).

En Panamá, existen pocos trabajos que evalúen el efecto que tiene la complejidad del hábitat sobre la estructura de la comunidad de peces arrecifales en áreas de acceso a la pesca. DominiciArosemena \& Wolf (2006), en un estudio realizado en Bahía Honda y alrededores, mencionan que los peces de arrecifes han tenido que utilizar hábitats rocosos que predominan en el Pacífico Oriental Tropical, resaltan la importancia de estos ambientes en la zona costera e indica que estos mantienen una estrecha conectividad con el PNC, en las diferentes etapas de desarrollo de los peces. Otro estudio realizado por Benfield et al., (2008), donde compara los ensambles de las comunidades de peces en arrecifes y comunidades coralinos en el Golfo de Panamá, indica que la 
contribución de los arrecifes rocosos tropicales a la diversidad de peces no debe pasarse por alto especialmente en regiones biogeográficas donde los arrecifes de coral están empobrecidos.

En el caso de la ensenada de Pixvae, la comunidad pesquera del área está interesada en la exclusión de las redes de enmalle de sus caladeros de pesca, pues mantienen una pesca tradicional con línea y anzuelo, es así como solicitaron a la Autoridad de los Recursos Acuáticos de Panamá se tomase esta medida, por lo cual este trabajo caracteriza los fondos asociados a los caladeros de pesca como insumo para apoyar la declaratoria de una zona de comanejo en la Ensenada.

\section{MATERIALES Y METODOS}

Área de estudio: Pixvae es un corregimiento que pertenece al distrito de Las Palmas, considerada una de las principales áreas de Influencia del Parque Nacional Coiba, y uno de los puntos más cercanos con acceso a este Parque. Es un caserío que se extiende aproximadamente por un kilómetro, limitado al este por un sistema de manglar y al oeste por la ensenada de Pixvae, donde viertes sus aguas los ríos Pixvae y La Mona, para cuyas cuencas se ha reportado un porcentaje de cobertura vegetal de $73 \%$ y $47 \%$, respectivamente (Veliela et al. 2014). Ambos ríos forman en su desembocadura un pequeño estuario cubierto de manglares donde ocurren suficientes trasformaciones de la materia orgánica para mantener la calidad de las aguas costeras de la ensenada de Pixvae (Valiela et al., 218).

La zona costera presenta una geología compleja, pendientes elevadas y suelos poco profundos, con una pendiente máxima de $9.8 \%$ para el río Pixvae y $7.1 \%$ para el rio la Mona (Castroviejo \& Ibáñez, 2005; Valiela et al., 2014). Los materiales geológicos subyacentes son altamente heterogéneos espacialmente, con basaltos fracturados y diabases volcánicas, esquistos, gabros y lutitas marinas y aluviales y areniscas (Valiela et al., 2013) (Figura 1).

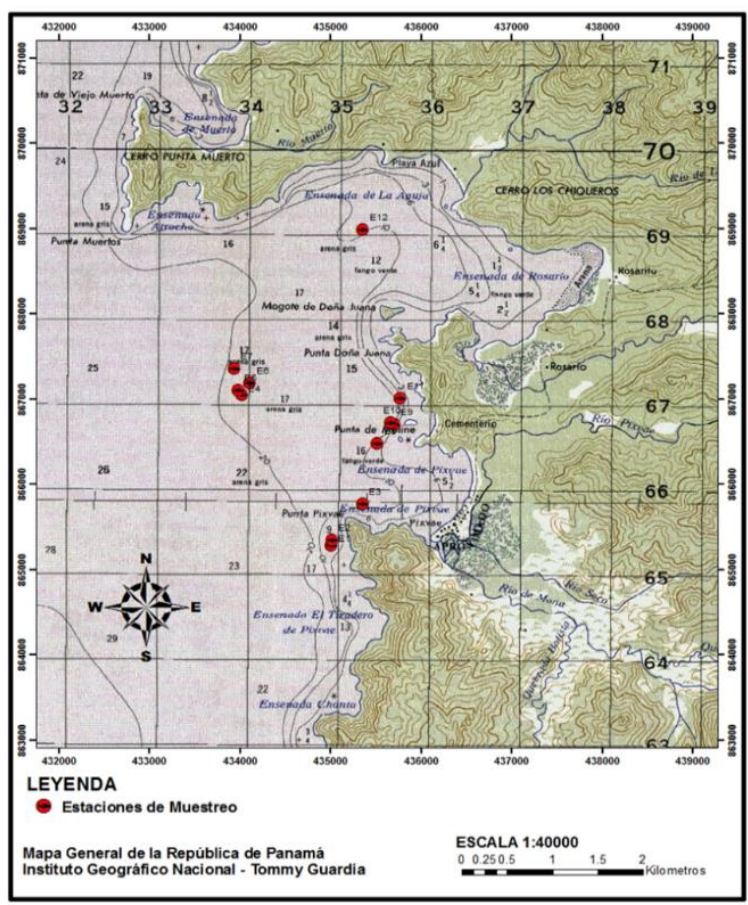

Figura. 1. Área de estudio, los puntos rojos indican los sitios de muestreo asociados a caladeros de pesca de la costa de Pixvae, Pacífico de Panamá. 
Para las evaluaciones y muestreos de los fondos se consideraron los principales caladeros utilizados por pescadores artesanales de la comunidad de Pixvae estos fueron georreferenciados con GPS, caracterizados mediante ecosondas e inmersiones con equipos de buceo autónomo; esta práctica se realizó durante la temporada seca (marzo a mayo 2015) y durante la lluviosa (agosto 2016).

Caracterización del hábitat: Para la composición del fondo (cobertura de los principales grupos morfofuncionales), se utilizó la técnica de videos transectos con cámaras digitales de video de alta resolución, para lo cual se filmó el substrato perpendicularmente al fondo, en un transecto de 50 m de largo (Garza-Pérez, 2011, modificado de Aronson \& Swanson, 1997). En cada transecto se estimó la profundidad con un profundímetro estándar de buceo, en tres puntos del transecto (inicio, en medio y final), se utilizó el valor promedio para cada sitio de buceo. Se estimó el número de oquedades presentes, en cada zona de muestreo, independientemente del tamaño de las rocas.

\section{Análisis de los datos}

Composición y cobertura de sustrato: Con el programa Coral Point Count con extensiones de Excel - V4.1 (CPCE - V4.1) Kohler \& Gill (2006), se tomaron 40 imágenes de cada video transecto, a cada una de estas se le montó un marco o cuadrante de $0.60 \mathrm{~m}^{2}$, donde se establecieron 12 puntos (480 puntos en total por transecto) y se identificó el tipo de fondo.

Para los porcentajes de cobertura se definieron 6 grupos morfofuncionales: Coral, equinodermo, esponja, alga, roca y arena. La cobertura de cada grupo se estimó según la ecuación: $\mathrm{C}=(\mathrm{NP} / 480) \times 100$, donde $\mathrm{C}$ es el porcentaje de cobertura de cada grupo morfofuncional y NP es el número de puntos observados en las 40 imágenes de cada video-transecto. Datos que fueron arrojados por el programa, además del índice de diversidad de Shannon (H') y el de dominancia de Simpson (D), para los bentos.

Complejidad topográfica: Se calculó para cada transecto, mediante el posicionamiento cuidadoso de una cadena $(20 \mathrm{~m})$, a partir del punto cero $(0)$ del transecto de $50 \mathrm{~m}$; se toma la distancia de la cadena siguiendo el contorno de fondo (d) y se divide por la longitud de la cadena (f), la relación obtenida a la inversa, se toma como un valor de complejidad topográfica (CT), siendo cero (0) completamente plano y uno (1) máxima complejidad topográfica.

$$
C T=1-\left(\frac{d}{f}\right)
$$

Complejidad estructural del hábitat: Se evaluó mediante análisis de clasificación "Cluster", tomando el porcentaje cobertura de los distintos grupos morfofuncionales, la profundidad, número de oquedades y la CT. El análisis se realizó con el paquete estadístico PAST y se aplicó la distancia Euclidiana mediante el método de agrupamiento de Ward. Para mayor afinidad, la información se ordenó mediante un Escalamiento Multidimensional no métrico (nMDS), las proximidades (similitud-disimilitud), ayudaron a definir los tipos de ambientes asociados a los caladeros.

Estructura de la comunidad íctica: La composición especies y abundancia de peces conspicuos fueron descritas mediante el método de censos visuales por medio de buceo autónomo, 
(Alzate et al., 2014). Para esto, se utilizaron los transectos ya establecidos en la descripción de los fondos (50 m de longitud) por $5 \mathrm{~m}$ de ancho (Samoilys \& Carlos, 2000; Galván-Villa et al., 2010).

Los censos se realizaron entre las 08:00 - 16:00 horas, los organismos encontrados fueron identificados hasta nivel de especie (Robertson \& Allen, 2015)

Diversidad: Se calculó mediante el índice de Shannon-Wiener H' $\left(\log _{10}\right)$, el índice proporciona información sobre la comunidad al considerar tanto el número de especies como la abundancia de cada una en los diferentes sitios muestreados.

$$
H^{\prime}=-\sum_{i=1}^{S}\left(\frac{n i}{N}\right) \log 10\left(\frac{n i}{N}\right)
$$

Equidad: Se evaluó con el índice de Pielou. El valor máximo se alcanza cuando todas las especies son igualmente abundantes, por lo tanto, valores altos cercanos a uno equivalen a condiciones de uniformidad en la comunidad y valores bajos, cercanos a cero indican dominancia de algunas especies.

$$
J=H^{\prime} / \ln (S)
$$

Índice de Valor Biológico (IVB): propuesto por Sanders, una de las características de este índice es el balanceo entre la abundancia espaciotemporal de especies, a través de la asignación de puntos en términos de la abundancia numérica en cada muestra (estación), en el cual se considera como una especie dominante a la que muestre constancia en su abundancia y frecuencia de aparición (Loya-Salinas y Escofet 1990) y recomienda usar el 95\% de los individuos por muestra para eliminar datos carentes de información relevante.

\section{Análisis estadístico}

Para determinar si existían diferencias significativas de la abundancia y riqueza de especies, respecto a las características del hábitat, se aplicó un análisis de varianza de una vía (ANOVA); en los casos donde se dieron diferencias se utilizó la prueba de Tukey HSD; para abundancias según profundidad, se hicieron comparaciones múltiples. Para conocer si existen diferencias significativas en cuanto a la diversidad y equidad en los dos años de estudio (2015-2016), se realizó una prueba t. Para los análisis se utilizó el programa SPSS, se estimaron los intervalos al 95\% de confianza.

La afinidad de la asociación entre las especies de peces más abundantes y el hábitat, se realizó mediante un análisis de correspondencia canónica (Dominici-Arosemena \& Wolf, 2006).

\section{RESULTADOS}

Se realizaron 51 inmersiones con 58:02 horas de buceo, en las doce estaciones de la costa de Pixvae. La profundidad promedio de los doce transectos varió de 5 a $23 \mathrm{~m}$. La mayor complejidad topográfica (CT) se presentó en Isla Muela y el valor más bajo en Playa Gone, una playa arenosa que muestra cierta estabilidad con formaciones de corales masivos dispersos (Cuadro $1)$. 
Rev. Col. Ciencia. Vol. 2, no. 1. Octubre 2020 - marzo 2021. ISSN L 2710-7434 pp. 42-56

Cuadro 1. Características de las estaciones de muestreo asociada a los caladeros de pesca en la costa de Pixvae, E: Estación, CT: Complejidad topográfica, Prof. (Profundidad promedio) y OQ. (número de oquedades).

\begin{tabular}{llllllcc}
\hline E & \multicolumn{1}{c}{ Nombre } & Código & \multicolumn{2}{c}{ Coordenadas } & CT & P (M) & OQ. \\
\hline E1 & Bajo la Boya S & BLBS & 865285 & 435003 & 0.066 & 12 & 34 \\
E2 & Bajo la Boya & BLB & 865349 & 435013 & 0.126 & 8 & 36 \\
E3 & Playa Gone & PG & 865775 & 435351 & 0.025 & 5 & 17 \\
E4 & Isla Muela S & IMS & 867054 & 434017 & 0.234 & 10 & 42 \\
E5 & Isla Muela O & IMO & 867106 & 433981 & 0.295 & 12 & 32 \\
E6 & Isla Muela E & IME & 867196 & 434105 & 0.093 & 7 & 70 \\
E7 & Isla Muela N & IMN & 867358 & 433929 & 0.043 & 14 & 36 \\
E8 & Bajo Ruth & BR & 866480 & 435512 & 0.050 & 11 & 38 \\
E9 & Bajo Moline II & BM II & 866699 & 435697 & 0.224 & 8 & 40 \\
E10 & Bajo Moline & BM & 866725 & 435659 & 0.124 & 7 & 46 \\
E11 & Isla Moline & IM & 867013 & 435764 & 0.159 & 7 & 36 \\
E12 & Bajo Goyo & BG & 868989 & 435351 & 0.123 & 23 & 14 \\
\hline
\end{tabular}

\section{Composición y cobertura del sustrato}

Los porcentajes de cobertura entre periodos de muestreo se distribuyen así: roca de $50 \%$ a $60 \%$, arena y grava en un $18 \%$, coral duro de $12 \%$ a $8 \%$, algas de un $11 \%$ a un $7 \%$, coral negro se mantuvo en un $2 \%$, octocorales de $1.5 \%$ a un $0.70 \%$, esponjas se mantuvo en $1 \%$ y equinodermos presento una disminución de $1.20 \%$ a $0.60 \%$.

Los mayores porcentajes de cobertura de coral se registraron alrededor de Isla Muelita (E4 y E6), aportando de un 25 a 30\% de la cobertura. La mayor riqueza de corales duros se dio en la Bahía de Pixvae (E9: 10 especies; E10: 9 especies) y alrededores de Isla Muelita (E4, E5 y E6, con 7 especies en cada sitio). Los octocorales tuvieron presencia en la mayoría de los sitios, pero la mayor riqueza y cobertura se dio en Bajo la Boya (E1 y E2) (Figura 2, Cuadro 2).

El índice de diversidad de Shannon-Weiner (H') en los dos periodos (2015-2016) no mostró diferencias significativas ( $\mathrm{p}>0.05$ ). Sin embargo, fue evidente el deterioro de importantes arreglos coralinos en la transición de ambos periodos.

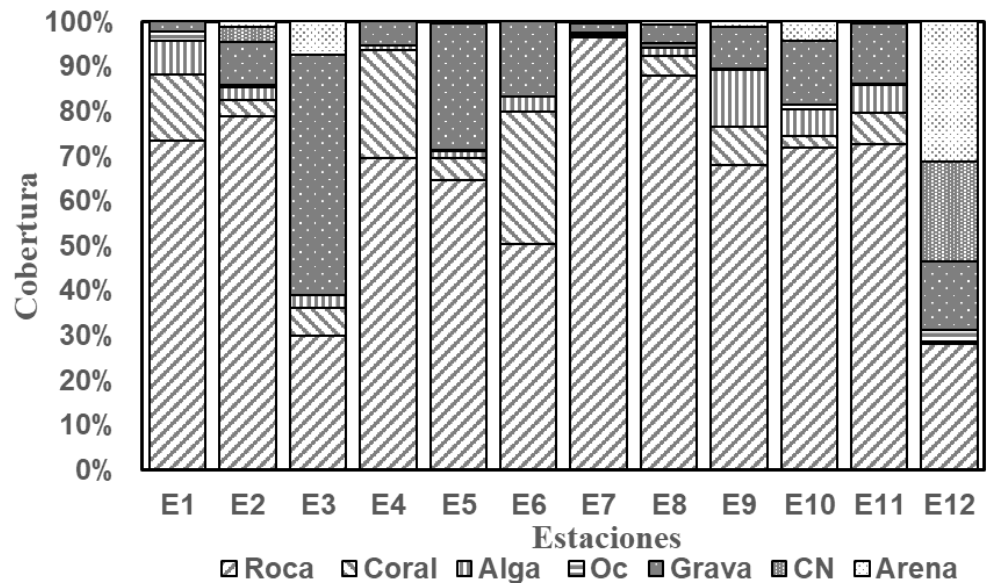

Figura 2. Porcentaje de cobertura de los principales grupos morfofuncionales, asociados a los caladeros de pesca en la costa de Pixvae. 
Rev. Col. Ciencia. Vol. 2, no. 1. Octubre 2020 - marzo 2021. ISSN L 2710-7434 pp. 42-56

Cuadro 2. Especies de organismos bentónicos, encontrados en las diferentes estaciones de muestreo en la costa de Pixvae, Golfo de Chiriquí.

\begin{tabular}{|c|c|c|c|c|c|c|c|c|c|c|c|c|}
\hline Especie & E1 & $\mathrm{E} 2$ & E3 & E4 & E5 & E6 & E7 & E8 & E9 & E10 & E11 & E12 \\
\hline \multicolumn{13}{|c|}{ Corales duros } \\
\hline Pocillopora damicornis & $\mathrm{x}$ & & $\mathrm{X}$ & $\mathrm{x}$ & $\mathrm{x}$ & $\mathrm{x}$ & $\mathrm{X}$ & & $\mathrm{x}$ & & $\mathrm{x}$ & \\
\hline Pocillopora elegans & $\mathrm{x}$ & $\mathrm{x}$ & $\mathrm{X}$ & $\mathrm{x}$ & $\mathrm{x}$ & $\mathrm{X}$ & $\mathrm{X}$ & $\mathrm{X}$ & $\mathrm{x}$ & $\mathrm{X}$ & $\mathrm{x}$ & \\
\hline Pocillopora eydouxi & & & & $\mathrm{x}$ & $\mathrm{x}$ & $\mathrm{X}$ & & & & & & \\
\hline Porites lobata & $\mathrm{X}$ & $\mathrm{x}$ & $\mathrm{X}$ & $\mathrm{x}$ & $\mathrm{x}$ & $\mathrm{X}$ & $\mathrm{X}$ & $\mathrm{x}$ & $\mathrm{X}$ & $\mathrm{x}$ & $\mathrm{X}$ & \\
\hline Porites panamensis & & $\mathrm{X}$ & $\mathrm{x}$ & $\mathrm{x}$ & $\mathrm{x}$ & $\mathrm{X}$ & & $\mathrm{X}$ & $\mathrm{x}$ & $\mathrm{x}$ & $\mathrm{x}$ & \\
\hline Gardinoseris planulata & & & & & & & & & $\mathrm{X}$ & & & \\
\hline Pavona chiriquensis & & & & & & & & & $\mathrm{x}$ & & $\mathrm{x}$ & \\
\hline Pavona clavus & $\mathrm{x}$ & $\mathrm{x}$ & $\mathrm{X}$ & $\mathrm{x}$ & $\mathrm{x}$ & & & $\mathrm{X}$ & $\mathrm{x}$ & $\mathrm{X}$ & $\mathrm{X}$ & \\
\hline Pavona gigantea & $\mathrm{x}$ & $\mathrm{X}$ & & & & & $\mathrm{X}$ & $\mathrm{X}$ & $\mathrm{x}$ & $\mathrm{X}$ & $\mathrm{X}$ & \\
\hline Pavona varians & & & & & & & & & $\mathrm{X}$ & $\mathrm{X}$ & $\mathrm{X}$ & \\
\hline Psammacora stellata & & & & $\mathrm{x}$ & $\mathrm{x}$ & $\mathrm{X}$ & & & $\mathrm{x}$ & & $\mathrm{X}$ & \\
\hline Tubastrea coccinea & & & & & & & & & & & & $\mathrm{x}$ \\
\hline \multicolumn{13}{|c|}{ Coral negro } \\
\hline Myriopathes panamensis & & $\mathrm{x}$ & & & & & & & & & & $\mathrm{x}$ \\
\hline \multicolumn{13}{|c|}{ Octocorales } \\
\hline Leptogorgia alba & $\mathrm{x}$ & $\mathrm{X}$ & & & $\mathrm{x}$ & & $\mathrm{X}$ & $\mathrm{X}$ & $\mathrm{x}$ & $\mathrm{X}$ & $\mathrm{x}$ & $\mathrm{x}$ \\
\hline Leptogorgia cuspidata & $\mathrm{x}$ & & & & & & & & & & & \\
\hline Pacifigorgia irene & $\mathrm{x}$ & $\mathrm{x}$ & & & & & & & & & & $\mathrm{x}$ \\
\hline Pacifigorgia rubicunda & & & & & & & & & & & & \\
\hline \multicolumn{13}{|c|}{ Equinodermos } \\
\hline Acanthaster planci & & & & & & & & & & $\mathrm{x}$ & & \\
\hline Pentaceraster cuminigi & & $\mathrm{x}$ & & & & & $\mathrm{X}$ & & $\mathrm{x}$ & & & \\
\hline \multicolumn{13}{|l|}{ Pharia pyramidatus } \\
\hline Diadema mexicanum & $\mathrm{x}$ & & & $\mathrm{x}$ & $\mathrm{x}$ & $\mathrm{X}$ & $\mathrm{x}$ & $\mathrm{x}$ & $\mathrm{x}$ & $\mathrm{x}$ & $\mathrm{x}$ & \\
\hline Isostichopus fuscus & & & & & & & $\mathrm{X}$ & & & & & \\
\hline Toxopneustes roseus & & & & & & & $\mathrm{X}$ & & & & & \\
\hline
\end{tabular}

\section{Complejidad estructural de hábitat}

El análisis "Clúster" de los 12 transectos basados en los atributos del hábitat (cobertura de los grupos morfofuncionales, número de oquedades, profundidad, complejidad topográfica) permitió identificar cuatro grupos de ambientes o fondos marinos (Figura 3). 
Rev. Col. Ciencia. Vol. 2, no. 1. Octubre 2020 - marzo 2021. ISSN L 2710-7434 pp. 42-56

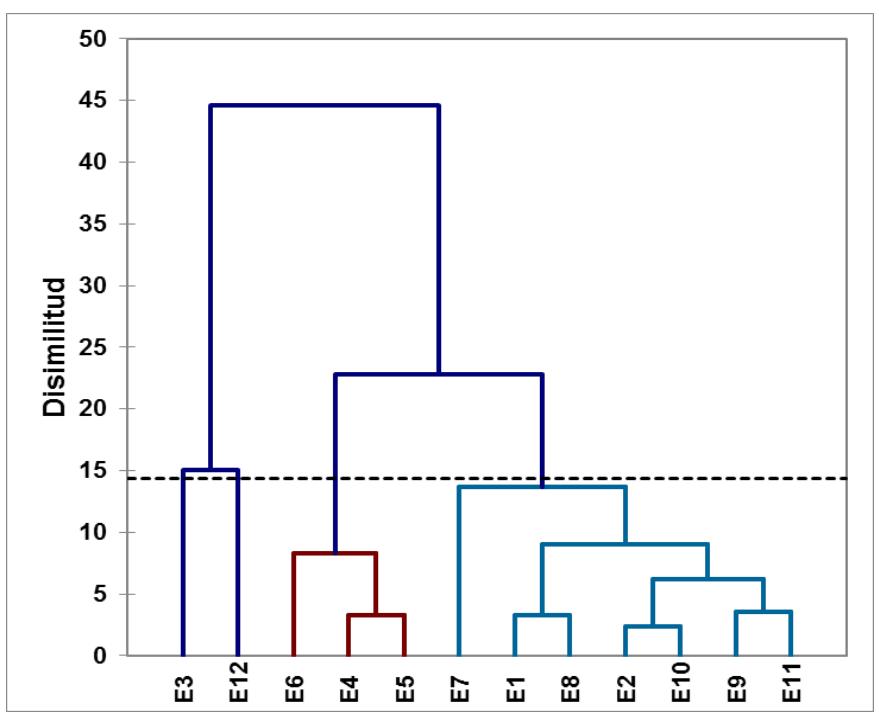

Figura 3. "Clúster" distancia Euclidiana - Método de Ward, de los atributos del hábitat para las 12 estaciones de muestreo (E1-E12) en la costa de Pixvae, Golfo de Chiriquí.

Ambiente de Fondos Rocosos (arrecifes rocosos): Son los fondos marinos dominantes en la costa de Pixvae, bajos de roca consolidada con superficies mayores a los $5 \mathrm{~m}$, estos ambientes están en profundidades que varían entre 7 a $14 \mathrm{~m}$, poblados por colonias de coral duro (ramificados y masivos) y octocorales; la roca se encuentran divididas por canales de arena-grava; su fauna la componen peces coralinos que aprovechan las grietas u oquedades para refugio de depredadores, este ambiente está dominado por peces de hábitos herbívoros principalmente, pero es común observar peces demersales, estadios juveniles de familias Lutjanidae (Lutjanus novemfasciatus, $L$. argentiventris), Serranidae (Cephalopholis panamensis, Epinephelus labribormis) y ocasionalmente peces nadadores activos (carángidos) que ingresan en gremios para alimentarse.

Ambientes rocosos de paredes y roca pequeña: Ambiente característico del sector sur, este y oeste de Isla Muelita (estaciones E4, E5 y E6); por ser uno de los sectores con más exposición oceánica presenta importantes arreglos coralinos, principalmente su cobertura, dominado por especies de la familia Pocillopora (Pocillopora damicornis, P. elegans y P. eydouxi), Poritidae (Porites lobata) y Agariciidae (Pavona clavus, P. gigantea); también cuenta con diversidad de peces coralinos de hábitos demersales y pelágicos, que incursionan al sitio para jornadas de alimentación; igualmente, este sitio es utilizado como estación de limpieza para especies transitorias de tortugas marinas (Eretmochelys imbricata y Chelonia mydas). Por otro lado, se observó que zonas de rocas pequeñas y disgregadas, son de importancia en el ciclo reproductivo de especies como el pez sargento (Abudefduf troschelii), las hembras adhieren los huevos a la roca y brindan cuidado parental, para evitar que peces coralinos se alimenten de estos.

Ambientes someros con formaciones de corales masivos: Este ambiente corresponde al sitio de Playa Gone (E3-PG), cercano a punta Pixvae, cuenta con un fondo compuesto de arenagrava (esqueleto de coral) principalmente y arreglos de corales masivos (Pavona clavus) a profundidades menores a $8 \mathrm{~m}$, con una fauna compuesta de peces juveniles de familias como Scaridae, Pomacentridae y Chaetodontidae, se aprecia poca densidad de peces y funciona como un 
sitio de criadero donde las especies pueden migrar a ambientes más profundos o hábitats cercanos, como arrecifes de corales masivos.

Ambientes de corales negros: Localizado en la bahía de Rosario (E12 - Bajo Goyo), es uno de los sitios más frecuentados por los pescadores del sector, cuenta con una profundidad promedio de $23 \mathrm{~m}$ y un fondo compuesto de arena-grava con importantes arreglos de corales negros que asemejan arbustos y que componen gran parte de la cobertura del transecto. Aquí confluyen grandes cardúmenes de peces (hemúlidos y lutjánidos, principalmente) y a diferencia de otros sitos, se observan grandes depredadores tope como pargos (Lutjanus novemfasciatus) y mero (Epinephelus quinquefasciatus), es un sitio muy productivo para la pesca del sector y sus atractivos lo potencian como un sitio de interés para el buceo recreativo.

La afinidad entre estos ambientes (similitud/disimilitud), que muestra el gráfico de Escalamiento Multidimensional no Métrico "nMDS" indican que los sitios cuentan con gran afinidad, principalmente los grupos 1 y 2 (que corresponde a ambientes rocosos y ambientes de paredes con roca pequeña), mientras que el grupo 3 que corresponde a fondos someros de arena con arreglos de corales masivos, muestra distancia, lo que describe como un ambiente diferente; al igual que el grupo 4 que corresponde a la E12 Bajo Goyo, uno de los sitos más profundos, que cuenta con un ambiente característico compuesto principalmente de corales negros (Figura 4).

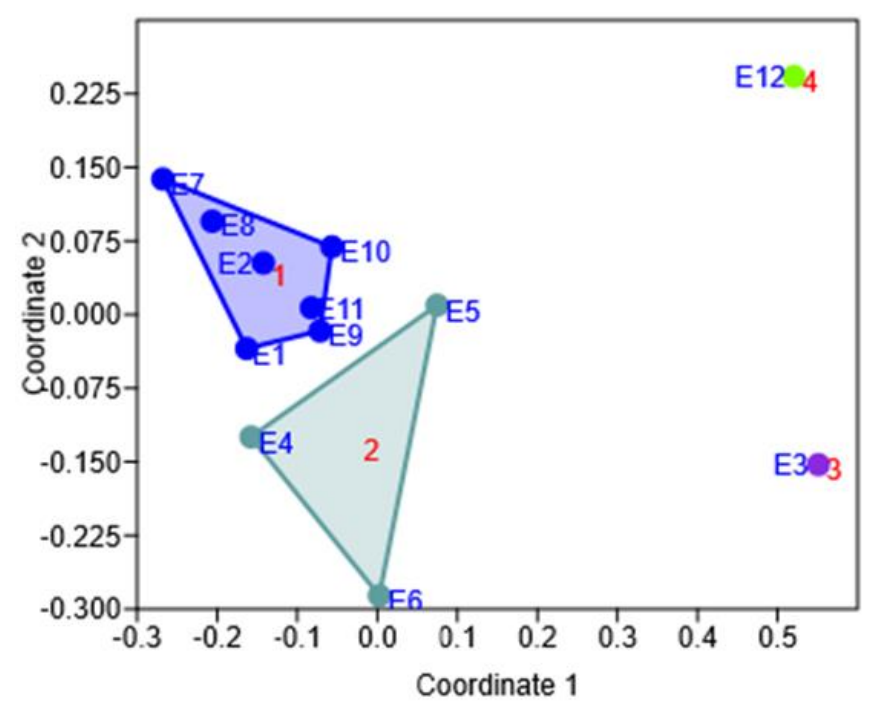

Figura 4. Escalamiento multidimensional no métrico (nMDS), muestra los patrones espaciales de afinidad de los sitios de muestreo en la costa de Pixvae.

El ANOVA de un factor muestra diferencias significativas entre los grupos, respecto al número de oquedades, el índice de Simpson's, la riqueza y abundancia. 
Rev. Col. Ciencia. Vol. 2, no. 1. Octubre 2020 - marzo 2021. ISSN L 2710-7434 pp. 42-56

Cuadro No. 3. Resultados de ANOVA de una vía y comparaciones múltiples entre grupos de ambientes en la costa de Pixvae.

\begin{tabular}{|c|c|c|c|}
\hline & $p$ & $\mathbf{F}$ & Comparaciones múltiples \\
\hline $\begin{array}{l}\text { Diversidad de sustrato (índice de } \\
\text { Simpson's) }\end{array}$ & 0.000 & 0.73 & grupo $1=$ grupo $2 \neq$ otros grupos \\
\hline Oquedades & 0.005 & 7.939 & grupo $1=$ grupo $2 \neq$ otros grupos \\
\hline Riqueza & 0.003 & 9.191 & grupo $1=$ grupo $2 \neq$ otros grupos \\
\hline Abundancia & 0.000 & 20.387 & grupo $1=$ grupo $2=$ grupo $3 \neq$ grupo 4 \\
\hline
\end{tabular}

\section{Análisis de la comunidad de peces}

Se contabilizaron un total de 3348 peces, agrupados dentro de 20 familias, 34 géneros y 50 especies. Las familias con mayor cantidad de especies fueron: Labridae (5), Haemulidae (5), Lutjanidae (4), Pomacentridae (4), Scaridae (4) y Serranidae (4). Los sitios E1 y E2 (Bajo la Boya) ubicados en Punta Pixvae y el sitio E10 (Bajo Moliné) localizado en la bahía de Pixvae, presentaron la mayor riqueza con 25 especies y los valores más bajos se registraron en el sitio E3 (Playa Gone) y E12 (Bajo Goyo), con 11 y 12 especies, respectivamente. Los demás sitos no muestrearon una marcada diferencia con rangos que van de 15 a 21 especies. Al realizar un análisis de la varianza se encontró diferencias significativas entre la riqueza de especies entre sitios localidades $(\mathrm{P}<0.05)$.

\section{Índice de Valor Biológico (IVB)}

De acuerdo con los resultados del IVB general, 10 especies fueron consideradas dominantes para el año 2015, Stegastes flavilatus (8.74\%), Canthigaster punctatissima (8.32\%), Abudefduf troschelii (7.31\%), Stegastes acapulcoensis (7.31\%), Haemulon maculicauda (7.14\%), Cephalopholis colonus (6.81\%) y Haemulon steindachneri (4.29\%). Mientas que para el 2016 fueron: Stegastes flavilatus (8.23\%), Canthigaster punctatissima (7.61\%), Stegastes acapulcoensis (6.60\%), Lutjanus argentiventris (5.63\%), Scarus ghobban (5.63\%), Lutjanus guttatus (5.19\%), Abudefduf troschelii (4.84\%), Chaetodon humeralis (4.22\%) y Haemulon maculicauda (4.18\%). Estas especies acumularon el $50 \%$ del puntaje total para ambos periodos, sin cambios marcados en la dominancia (Figura 5).
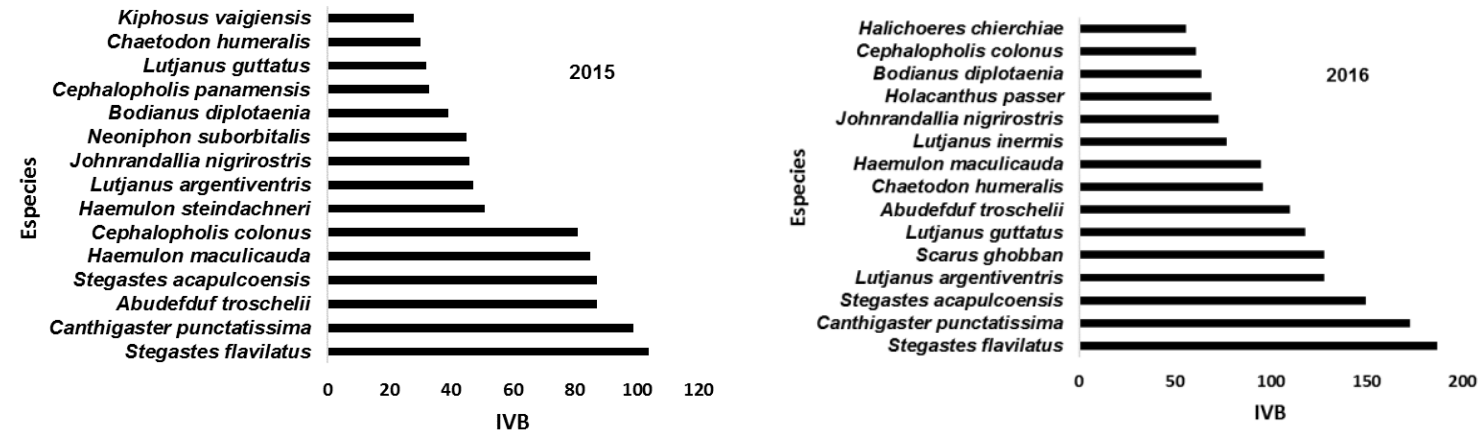

Figura 5. Especies de peces dominantes en la costa de Pixvae, de acuerdo con los valores del Índice de Valor Biológico. A) periodo 2015; B) periodo 2016. 
Rev. Col. Ciencia. Vol. 2, no. 1. Octubre 2020 - marzo 2021. ISSN L 2710-7434 pp. 42-56

\section{Análisis de correspondencia canónica (CCA)}

Al relacionar los atributos de la complejidad estructural del hábitat, con la presencia de especies de peces de mayor dominancia, se observa que especies como Lutjanus guttatus (LGU) y Chaetodon humeralis (CHU), están vinculados a ambientes profundos, como también a la presencia de corales negros y octocorales; por otra parte, en ambientes de roca consolidada o arrecifes rocosos de profundidad media $( \pm 15 \mathrm{~m})$, está estrechamente relacionado con la presencia de especies de peces coralinos como Haemulon maculidauda (HMA), Johnradalia nigrirostris (JNI), Cephalopholis colonus (CCO), Holacanthus passer (HPA) y Scarus ghobban (SGH). Mientras que la rugosidad, el número de oquedades y la cobertura de corales duros, estuvo relacionada con especies como Stegastes flavilatus (SFL), S. acapulcoensis (SAC), Lutjanus argentiventris (LAR) y Bodianus diplotaenia (BDI) (Figura 6).

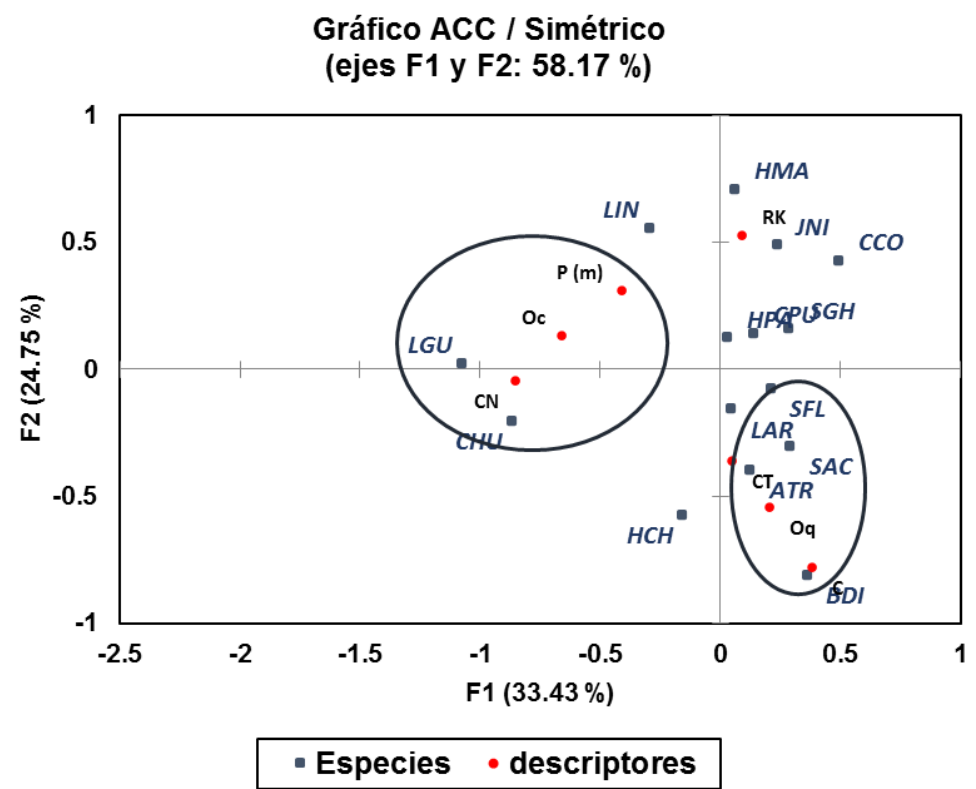

Figura 6. Análisis de correspondencia canónica (CCA) de peces conspicuos relacionados con los atributos del hábitat

\section{DISCUSIÓN}

Características del hábitat: Los resultados muestran que en la costa de Pixvae, los ambientes marinos asociados a los caladeros de uso artesanal, están constituidos principalmente de bajos rocosos con cobertura promedio de 65\% (ámbito 27.71-92.08); Se pudieron identificar cuatro grupos de ambientes o fondos, con base en los descriptores del hábitat, siendo los fondos arrecifales tipo rocoso (Grupo 1) los dominantes y presentan gran afinidad de atributos ecológicos de los fondos rocosos de paredes y rocas pequeñas (Grupo 2). Al analizar los índices ecológicos, muestra que ambientes rocosos asociados a Isla Muela (E5) y la bahía de Pixvae (E7, E8 y E9) son topográficamente más complejos y albergan una mayor diversidad de organismos bentónicos; sin embargo, la fauna bentónica de invertebrados móviles estuvo compuesta de equinodermos y se 
observaron importantes concentraciones en alrededores de Isla Muela. Graham et al., (2011), encontraron que la composición de la comunidad bentónica en el POT (Costa Rica, Panamá, Colombia y Ecuador), muestra cierto grado de variación con mayores densidades en zonas costeras de acceso a la pesca con relación a áreas marinas protegidas, consideran esta variación un efecto secundario de la sobrepesca, debido a que los macroinvertebrado son presas de grandes peces depredadores (meros, pargos) y langostas (Panulirus gracilis); la presencia de esta última, estuvo asociada a Isla Muela y Bajo Goyo. Por otra parte, este sustrato rocoso promueve el asentamiento de importantes arreglos coralinos y fauna asociada. Se registran 12 especies de coral escleractinio de las 23 especies descritas por Guzmán \& Breedy (2008), para el Pacifico Occidental de Panamá.

Estudios realizados en los golfos de Chiriquí y Panamá destacan la importancia de los fondos rocosos como hábitat para un sinnúmero de especies, principalmente por la disponibilidad de refugio y alimento, especialmente en áreas biogeográficas empobrecidas de arrecifes coralinos como es el caso del POT (Dominicci-Arosemena \& Wolf, 2006; Benfield, et al., 2008). La cobertura promedio de coral fue de $9.5 \%$ (ámbito 8-12) y estuvo compuesta principalmente por especies del género Pocillopora (>70\%). Ambientes de someros de arena con arreglos de corales masivos (Grupo 3) corresponde a un ambiente simple (0.025 complejidad topográfica) con escaza presencia de invertebrados móviles y corales blandos. En el caso de ambientes profundos dominados de corales negros (Grupo 4), muestra cobertura de $25 \%$, con arreglos que alcanzan hasta cerca de $1.5 \mathrm{~m}$, sus ramas permiten que se fijen bivalvos y en las bases rocosas se resguardan algunas langostas; presenta una menor riqueza de especies si se compara con los bajos rocosos; sin embargo, este fondo cubierto de vida propicia la abundancia de algunas especies peces. Gratwicke \& Speight (2005), al relacionar las variables que definen el hábitat, encuentra que la altura del fondo es un factor que incide en la abundancia de peces.

Análisis de la comunidad de peces: Este estudio encontró que la profundidad es un factor determinante en la abundancia de peces, Bajo Goyo (E12) mostró diferencias significativas en abundancia con relación a los demás sitios, se reportaron abundancia $>500$ individuos un promedio de 90.15, especialmente de la familia Lutjanidae (Lutjanus guttatus) y Haemulidae (Haemulon maculicauda), mostró diferencia significativa con relación a los demás sitios. Seguidamente, el sector de Bajo La Boya (E1 y E2) con 242 y 277 organismos, con promedios de 18.62 y 21.31 respectivamente, un aspecto importante de resaltar es que los ambientes de ambas localidades Bajo Goyo y la Boya, cuentan con presencia de corales negros, Gratwicke y Speight (2005) indican que un componente que influye en la abundancia de peces es la altura del fondo. Otro aspecto importante para destacar es que, en ambientes profundos de corales negros, se observaron depredadores topes $(>1 \mathrm{~m})$ como el Mero de profundidad del Pacífico (Epinephelus quinquefasciatus) y pargo negro (Lutjanus novemfasciatus),

La relación entre los atributos del hábitat y la presencia de peces dominantes indica que peces de importancia comercial como el grupo de los pargos, mantienen presencia en la mayoría de los sitios, especies como Lutjanus argentiventris, se encuentran en ambientes rocosos de poca profundidad, mientras que Lutjanus guttatus presenta importantes abundancias en ambientes profundos, indicando que existe gran disponibilidad de recursos en los diferentes sitios de pesca en la costa de Pixvae. Por otra parte, ambientes rocosos de la bahía de Pixvae sostienen un elenco diverso de grupos tróficos que incluye peces plactívoros, herbívoros, invertívoros móviles que muestran una amplia distribución espacial y se encuentran relacionados con la cobertura de coral. Según Glynn (2017), especies de la familia Scaridae desempeñan un rol de importancia en la repoblación de colonias de coral. También se observa que especies Abudefduf troschelii muestran 
gran relación con las oquedades de ambientes rocosos de paredes y roca pequeña, ya que utilizan estos sitos para la reproducción, aquí adhieren sus huevos en las oquedades que se forman entre rocas y brindan un cuidado parental ante los depredadores, principalmente peces coralinos como Microsphatodon dorsalis, Chaetodon humeralis y Holacanthus passer. De manera general, el elenco de peces conspicuos de la costa de Pixvae, se encuentra dominado por peces de coralinos de tamaño pequeño, son pocos los avistamientos de depredadores tope, los cuales están relacionados a fondos profundos de corales negros, en más de 50 inmersiones solo se avistaron dos tiburones punta blanca (Triaenodon obesus), asociado a los sectores más alejados de la costa. Según Sala et al., (2004), la ausencia de depredadores topes es uno de los primeros indicativos de sobrepesca y casos como este es común a lo largo de las costas del Pacifico Oriental Tropical, por lo que son necesario contar con herramientas de manejo que ordenen la actividad de pesca que aquí se desarrolla, principalmente por la conectividad entre ambientes y sus recursos con el Parque Nacional Coiba.

\section{CONCLUSIÓN}

Los ambientes coralinos de la costa de Pixvae se componen de bajos rocosos en un $60 \%$, estos bajos permiten el asentamiento de importantes arreglos de corales escleractino principalmente de la familia Milleporidae y están estrechamente relacionados con la riqueza de peces coralinos del sector, mientras que ambientes profundos mayores a $20 \mathrm{~m}$ están dominados por la especie de coral negro (Myriopathes panamensis) está relacionado a la abundancia de pargo mancha (Lutjanus guttatus), roncadores y presencia de depredadores tope como mero (Epinephelus quinquenfasciatus) y pargo dientón (Lutjanus novemfasciatus). Estas comunidades coralinas deben ser atendidas, en función de dar protección especial, tal y como lo solicita la comunidad de Pixvae, al pedir la exclusión del uso de trasmallos en la zona.

\section{AGRADECIMIENTOS}

Se agradece a Conservación Internacional (CI), por el apoyo financiero para el desarrollo del proyecto y a la comunidad de Pixvae.

\section{REFERENCIAS}

Alzate, A, Zapata, F. \& Giraldo, A. (2014). A comparison of visual and collection-based methods for assessing community structure of coral reef fishes in the Tropical Eastern Pacific. Rev. Biol. Trop., 62 (Suppl. 1), 359-371.

Aronson, R.B. \& Swanson, D.W. (1997). Video surveys of coral reefs: uni and multivariate applications. Proc. 8th Int. Coral Reef Sym., 2, 1923-1926.

Benfield, S., Baxter, L., Guzman H. \& Mair, J. (2008). A comparison of coral reef and coral community fish assemblages in Pacific Panamá and environmental factors governing their structure. Journal of the Marine Biological Association of the United Kingdom, 88 (7), 1331-1341.

Castroviejo, S. e Ibáñez, A. (2005). Estudios sobre la biodiversidad de la región de Bahía Honda (Veraguas, Panamá). Consejo Superior de Investigaciones Científicas, España. 
Rev. Col. Ciencia. Vol. 2, no. 1. Octubre 2020 - marzo 2021. ISSN L 2710-7434 pp. 42-56

Dominici-Arosemena, A \& Wolff, M. (2006). Reef fish community structure in the Tropical Eastern Pacific (Panamá): living on a relatively stable rocky reef environment. Helgol. Mar. Res., 60, 287-305.

Ferreira C., E.L., Gonclaves, J. E.A. \& Coutinho, R. (2000). Community structure of fishes and habitat complexity on a tropical rocky shore. Environmental Biology of Fishes, 61, 353369.

Friedlander, A.M. \& Parrish, J.D. (1997). Habitat characteristics affecting fish assemblages on a Hawaiian coral reef. Journal of Experimental Marine Biology and Ecology, 224, 1-30.

Galván-Villa, C. M., Arreola-Robles, J. L., Ríos-Jara, E. \& Rodríguez-Zaragoza, F. (2010). Reef fish assemblages and their relation with the benthic habitat of Isabel Island, Nayarit, México. Revista de Biología Marina y Oceanografía, 45 (2), 311-324.

Garza-Pérez J.R. (2011). Evaluación de comunidades bentónicas arrecifales. Guía de campo y laboratorio. PAPIME, DGAPA-UNAM, 25pp.

Glynn, P. W. (2017). History of Eastern Pacific Coral Reef Research. Coral reef of the Tropical Eastern Pacific persistence and loss in a dynamic environment. Coral reef of the world 8, Springer, 1-38p.

Graham, J.E., Banks, S.A., Bessudo, S., Cortés, J., Guzmán, H.M., Henderson, S., Martinez, C., Rivera, F., Soler, G., Ruiz, D. \& Zapata, F.A. (2011). Variation in reef fish and invertebrate communities with level of protection from fishing across the Eastern Tropical Pacific seascape. Global Ecology and Biogeography, 20, 730-743.

Gratwicke, B. \& Speight, M.R (2005). The relationship between fish species richness, abundance and habitat complexity in a range of shallow tropical marine habitats. Journal of Fish Biology, 66, 650-667

Guzmán, H.M., y Breedy, O. (2008). Distribución de la diversidad y estado de conservación de los arrecifes coralinos y comunidades coralinas del Pacífico occidental de Panamá (Punta Mala - Punta Burica). First ed. Arlington, Virginia: The Nature Conservancy.

Kohler, K.E. \& Gill, S.M. (2006) Coral Point Count with Excel Extensions (CPCe): A Visual Basic Program for the Determination of Coral and Substrate Coverage Using Random Point Count Methodology. Computers and Geosciences, 32, 1259-1269.

Loya-Salinas, D.H. y Escofet, A. (1990). Aportaciones al cálculo del índice de valor biológico (Sanders 1960). Ciencias Marinas, 16(2), 97-115.

Pauly, D., Christensen, V., Dalsgaard, J., Froese, R. \& Torres, F. (1998). Fishing down marine food webs. Science, 279, 860-863.

Ramírez-Ortiz, G., Calderón-Aguilera, L.E., Reyes-Bonilla, H., Ayala-Bocos, A. Hernández, L. Rivera-Melo, F. López-Pérez A. \& Dominici-Arosemena. A. (2017). Functional diversity 
Rev. Col. Ciencia. Vol. 2, no. 1. Octubre 2020 - marzo 2021.

of fish and invertebrates in coral and rocky reefs of the Eastern Tropical Pacific. Marine Ecology, 38, e12447.

Roberts, C.M. (1995). Effects of Fishing on the Ecosystem Structure. Conservation Biology, 9 (5), 988-995.

Roberts CM, Bohnsack, J.A., Gell, F., Hawkins, J.P. \& Goodridge, R. (2001). Effects of marine reserve on adjacent fisheries. Science, 294, 1920-1923.

Robertson, D.R. \& Allen, G.R. (2015). Shorefishes of the Tropical Eastern Pacific: online information system. Versión 2.0 Smithsonian Tropical Research Institute, Balboa, Panamá.

Sala, E., Aburto-Oropeza, O, Reza, M., Paredes, G. \& López-Lemus, L.G. (2004). Fishing Down Coastal Food Webs in the Gulf of California, Fisheries, 29:3, 19-25.

Salas S, Chuenpagdee, R., Seijo, J.C. \& Charles, A. (2007). Challenges in the assessment and management of small-scale fisheries in Latin America and the Caribbean. Fisheries Research, 87, 5-16.

Samoilys, M.A. \& Carlos, G. (2000). Determining methods of underwater visual census for estimating the abundance of coral reef fishes. Environmental Biology of Fishes 57, 289304.

Tokeshi, M. \& Arakaki, S. (2012). Habitat complexity in aquatic systems: fractals and beyond. Hydrobiologia, 685, 27-47.

Valiela, I., Pascual, J., Giblina, A, Barth-Jensena, C., Martinetto, P., Ottera, M., Stone, T, Tucker, J., Bartholomew, M. \& Vianad, I.G. (2018). External and local controls on land-sea coupling assessed by stable isotopic signatures of mangrove producers in estuaries of Pacific Panama. Marine Environmental Research 137, 133-144

Valiela, I., Bartholomew, M., Giblin, A., Tucker, J., Harris, C., Martinetto, P., M. Otter, Camilli, L. \& Stone, T., (2014). Watershed deforestation and down-estuary transformations alter sources, transport, and export of suspended particles in Panamanian mangrove estuaries. Ecosystems 17, 91-111.

Valiela, I., Giblin, A., Barth-Jensen, C., Harris, C., Stone, T., Fox, S. \& Crusius, J., (2013). Nutrient gradients in Panamanian estuaries: effects of watershed deforestation, rainfall, upwelling, and within-estuary transformations. Mar. Ecol. Prog. Ser. 482, 1-15.

Vega, A. J, Robles P., Y.A. y Maté, J.L. (2016a). La pesca artesanal en el Parque Nacional Coiba y zona de influencia. Biología y pesquería de sus principales recursos, con recomendaciones de manejo. Fundación MarViva, Ciudad de Panamá, Panamá, 67pp.

Vega A.J., Maté, J. \& Robles P., Y. A. (2016b). First Report of Reproductive Aggregations for Pacific Red Snappers Lutjanus peru (Nicholson y Murphy, 1992) and Spotted Rose Snapper L. guttatus (Steindachner, 1869) in the Coiba National Park, Pacific of Panama. Proceedings of the 68th Gulf and Caribbean Fisheries Institute. (68):112-117. 